OPEN ACCESS

Edited by:

Marie-France Penet, Johns Hopkins University,

United States

Reviewed by:

Gunnar Kristensen,

Oslo University Hospital, Norway Anna Myriam Perrone,

Sant'Orsola-Malpighi Polyclinic, Italy

${ }^{*}$ Correspondence:

Yunlong Yue

yueyunlong@bjsith.cn

${ }^{\dagger}$ These authors have contributed equally to this work and

share first authorship

Specialty section: This article was submitted to

Cancer Imaging and Image-directed Interventions,

a section of the journal

Frontiers in Oncology

Received: 12 October 2021 Accepted: 20 December 2021

Published: 17 January 2022

Citation:

Cui T, Shi F, Gu B, Jin Y, Guo J, Zhang C, Ren J and Yue Y (2022) Peritumoral Enhancement for the Evaluation of Myometrial Invasion in Low-Risk Endometrial Carcinoma on Dynamic Contrast-Enhanced MRI.

Front. Oncol. 11:793709. doi: 10.3389/fonc.2021.793709

\section{Peritumoral Enhancement for the Evaluation of Myometrial Invasion in Low-Risk Endometrial Carcinoma on Dynamic Contrast-Enhanced MRI}

\author{
Tingting Cui ${ }^{1 \dagger}$, Feng Shi ${ }^{2 \dagger}$, Bei Gu ${ }^{3}$, Yanfang Jin ${ }^{1}$, Jinsong Guo ${ }^{1}$, Chao Zhang ${ }^{1}$, \\ Jie Ren ${ }^{1}$ and Yunlong Yue ${ }^{1 *}$ \\ ${ }^{1}$ Department of MR, Beijing Shijitan Hospital, Capital Medical University, Beijing, China, ${ }^{2}$ Department of Pathology, Beijing \\ Shijitan Hospital, Capital Medical University, Beijing, China, ${ }^{3}$ Department of Obstetrics and Gynecology, Beijing Shijitan \\ Hospital, Capital Medical University, Beijing, China
}

Objectives: To explore the clinical value of subendometrial enhancement (SEE), irregular thin-layered peritumoral early enhancement (ITLPE) and focal irregular peritumoral early enhancement (FIPE) on dynamic contrast-enhanced magnetic resonance imaging (DCEMRI) for myometrial invasion in patients with low-risk endometrial carcinoma.

Methods: Seventy-seven patients with low-risk endometrial carcinoma who preoperatively underwent DCE-MRI were included. Two radiologists independently evaluated and recorded the occurrences of SEE, ITLPE and FIPE on DCE-MRI in all patients. Interobserver agreement was calculated between the two radiologists, and the relationships between SEE, ITLPE, FIPE, and myometrial invasion were analyzed based on histologic findings. For statistically significant findings, the sensitivity and specificity were calculated, and the differences in myometrial invasion evaluations were analyzed. For those with no statistical significance, images were compared with the histopathologic sections.

Results: Inter-observer agreement was good $(k=0.80 ; 95 \% \mathrm{Cl}, 0.577-0.955)$ for SEE, and very good $(k=0.88 ; 95 \% \mathrm{Cl}, 0.761-0.972)(k=0.86 ; 95 \% \mathrm{Cl}, 0.739-0.973)$ for ITLPE and FIPE. After consensus, SEE was identified in 12/77 (15.6\%) patients; ITLPE and FIPE were found in 53/77 (68.8\%) and 30/77 (39.0\%) patients, respectively. SEE and ITLPE were significantly correlated with myometrial infiltration $(P=0.000)$, but FIPE were not $(P=0.725)$. The sensitivity and specificity of SEE and ITLPE for myometrial invasion in patients with low-risk endometrial carcinoma were 95.0 and $52.9 \%$, and 85.0 and $88.0 \%$, respectively. The area under the curve (AUC) of SEE and ITLPE for myometrial invasion were $0.740(95 \% \mathrm{Cl}, 0.584-0.896)$, and $0.866(95 \% \mathrm{Cl}, 0.763-$ $0.970)$, respectively. The sensitivity and specificity were statistically different between SEE and ITLPE for the detection of myometrial invasion ( $P=0.031,0.016)$. According to the comparison between FIPE and histopathologic findings, the irregular 
endomyometrial junction was found in 30/77 (38.9\%) cases, 24/30 (80.0\%) with myometrial infiltration and 6/30 (20.0\%) cases without myometrial infiltration.

Conclusions: FIPE was the irregular endomyometrial junction. It can be found in patients with or without myometrial infiltration and may lead to the overestimation of myometrial invasion by SEE on DCE-MRI. ITLPE presented high diagnostic performance and specificity for myometrial invasion in patients with low-risk endometrial carcinoma.

Keywords: magnetic resonance imaging, endometrial carcinoma, uterus, dynamic contrast-enhanced imaging, risk classification

\section{INTRODUCTION}

Endometrial carcinoma is the most common gynecologic malignancy in women worldwide. The tumor has a global incidence of 417, 000 new cases and 97, 000 deaths in 2020 (1). The incidence of this disease in younger women has been increasing in parallel with increases in obesity, nulliparity, and polycystic ovarian syndrome (2-5). Approximately 5-30\% of all reported endometrial carcinoma cases were diagnosed in younger women (6-8). For those patients, fertility preservation should be taken into consideration when deciding optimal management. Progestogen therapy might be an option in patients with low-grade endometrioid carcinoma in the absence of any myometrial invasion based on medical imaging $(9,10)$. Generally, the younger women diagnosed with endometrial carcinoma usually have a better outcome, because the tumor tends to present with favorable disease features, such as a favorable histologic subtype, with a lower grade lesion and minimal or absent myometrial invasion (11-16). Endometrioid adenocarcinoma (favorable histologic subtype), G1 and G2 (lower tumor grade), Stage IA (no or less than half myometrial invasion) are at low risk according to the European Society for Medical Oncology (ESMO) clinical practice guideline for risk classification of endometrial cancer (17). Information about histologic subtype and tumor grade can be acquired by curettage; however, curettage does not give information on myometrial invasion. Therefore, myometrium infiltration assessments are needed preoperatively in patients with low-risk endometrial carcinoma so that fertility-sparing progestogen therapy can be prescribed in these patients.

Magnetic resonance imaging (MRI) is considered to be a reliable modality for the evaluation of myometrial invasion of endometrial carcinoma for its excellent soft tissue contrast (18). Myometrial invasion is often assessed by previously published standards as follows: an interrupted junctional zone (JZ) on T2weighted MR images and subendometrial enhancement (SEE) on dynamic contrast-enhanced (DCE) images. According to previous studies, low signal intensity $\mathrm{JZ}$ is the boundary between the endometrium and myometrium based on T2-

Abbreviations: SEE, subendometrial enhancement; DCE-MRI, dynamic contrastenhanced magnetic resonance imaging; ITLPE, irregular thin-layered peritumoral early enhancement; FIPE, focal irregular peritumoral early enhancement; ESMO, European Society for Medical Oncology; JZ, junctional zone; DWI, diffusionweighted imaging. weighted MR images, and SEE is the thin-layered enhancement between the endometrium and myometrium on DCE images (19, 20). However, JZ may be poorly visible due to age, menstrual cycle, acyeterion or hormone mimetics. Therefore, the diagnostic accuracy of myometrial invasion is lower if done only with T2weighted images $(21,22)$. Nowadays, the diagnostic efficiency of myometrial invasion has been improved by DCE-MRI and diffusion-weighted imaging (DWI). In young women with endometrial cancer who want fertility-sparing progestogen therapy, DCE-MRI has been found superior to DWI in excluding myometrial invasion (23). With temporal and spatial resolution improvements, the sensitivity of SEE on DCE-MRI for myometrial invasion has ranged from 70 to $90 \%$, but the specificity can be as low as $30 \%(24-26)$. The SEE is not easily detected in premenopausal patients, except during the proliferative phase of the menstrual cycle (27).This may result in a lower specificity for myometrial invasion assessments. Therefore, improving the specificity of DCE-MRI in detecting myometrial invasion in patients with low-risk endometrial cancer may be a new challenge.

Irregular thin-layered peritumoral early enhancement (ITLPE) and focal irregular peritumoral early enhancement (FIPE) were described firstly by Fujii et al. as the detailed information about the interface between endometrial carcinoma and myometrium by DCE-MRI. ITLPE was found to be related to myometrial invasion, although FIPE as a controversial finding for myometrial infiltration $(26,28)$. To the best of our knowledge, there are only a few publications about the diagnostic performance of ITLPE in assessing myometrial invasion in patients with low-risk endometrial carcinoma and further study of FIPE.

In this study, we aimed to assess the relationship between SEE, ITLPE, FIPE, and myometrial invasion and evaluated the diagnostic performance of SEE and ITLPE for myometrial invasion in patients with low-risk endometrial carcinoma. In addition, we compared FIPE with histopathologic findings.

\section{MATERIAL AND METHODS}

\section{Study Population}

After being approved by the Institutional Review Board and obtaining informed consents, a total of 96 consecutive patients pathologically diagnosed as endometrioid carcinoma were included 
at our hospital from June 2017 to March 2021. All patients underwent preoperative pelvic DCE-MRI. According to the ESMO clinical practice guidelines for endometrial carcinoma, patients with low-risk endometrioid carcinoma (2009 FIGO stage IA, G1/G2) were enrolled. The exclusion criteria were the following: 1) patients who were diagnosed by biopsy $(n=8) ; 2)$ patients who received tumor-related treatments (radiotherapy or chemotherapy) before the pelvic DCE-MRI scan $(\mathrm{n}=4) ; 3)$ the time between DCEMRI and surgery was $>30$ days $(n=3) ; 4)$ poor image quality $(\mathrm{n}=$ 4). Seventy-seven patients (40-77 years; mean 60 years) were eventually included in the study.

\section{MRI Protocol}

MR examination was performed with a $1.5 \mathrm{~T}$ MR scanner (Ingenia, Philips Healthcare, The Netherlands) using a 32channel phased-array body coil. All patients were asked to fast at least $4 \mathrm{~h}$ before the MRI examination. A series of MR sequences were performed: 1) sagittal T2-weighted imagingturbo spin-echo (T2WI-TSE); 2) axial T2WI-TSE; 3) axial T1weighted imaging (T1WI)-mDIXON; and 4) axial diffusionweighted imaging (DWI). Subsequently, DCE-MRI with a flip angle of $15^{\circ}$ was acquired. At the second dynamic, $0.2 \mathrm{mmol} / \mathrm{kg}$ of contrast agent (Gadopentetate Dimeglumine Injection, CONSUN) was administered intravenously at a rate of $2.0 \mathrm{ml} / \mathrm{s}$ and followed by the same amount of $0.9 \%$ saline flush; Twentyfive dynamics were obtained consecutively, with a temporal resolution of $7.8 \mathrm{~s}$, and the acquisition time was $196 \mathrm{~s}$. MRI sequences and parameters are shown in Table $\mathbf{1}$.

\section{Image Analysis}

Image analysis was performed by two radiologists (with 20 and 25 years of experience in pelvic MRI, respectively) who were unaware of the depth of myometrial invasion (no myometrial invasion, tumor confined to the endometrium; superficial myometrial invasion, invading $<50 \%$ of the myometrium; and deep myometrial invasion, invading $>50 \%$ of the myometrium), tumor grade and surgical findings, except for the general diagnosis of endometrioid carcinoma. They independently evaluated and recorded occurrences of SEE, ITLPE and FIPE on DCE-MRI. Any discrepancy was resolved by consensus. Based on DCE-MRI, SEE was treated as a thin enhancement layer between the endometrium and myometrium (Figure 1), and was regular and smooth. According to the previous report (26), ITLPE was defined as an irregular thin-layered enhancement of the peritumoral area on early DCE images (Figure 2), and FIPE was the focal irregular enhancement of the peritumoral area, protruding toward the uterine cavity on early DCE images (Figures 3A, B).

\section{Histologic Analysis}

All of the 77 patients underwent hysterectomy and bilateral adnexectomy with or without pelvic or para-aortic lymph node dissection. Histopathologic information, namely, histologic subtype, tumor grade, and depth of myometrial invasion, was available for all patients. The cases where FIPE were detected on images were compared with the histopathologic findings, and the histopathologic sections were reviewed by the same pathologist. The criteria for myometrial invasion and irregular endomyometrial junction followed previously published standards $(29,30)$. A diagnosis of myometrial invasion could be made when neoplastic epithelial cells were surrounded by myometrium without intervening endometrial stroma. Also, myometrial invasion could also be diagnosed when jagged infiltrative contour and traditional desmoplastic stromal reaction were present. The irregular endomyometrial junction was defined as an endomyometrial interface with one or more undulations that measured not less than 2 to $3 \mathrm{~mm}$ in magnitude.

\section{Statistical Analysis}

Statistical analysis was performed with SPSS software (Version 22.0). Interobserver agreement between the two radiologists was calculated by the weighted Cohen's kappa; the $k$ value of $0.81-1.00$ indicated very good agreement, $0.61-0.80$ indicated good, $0.41-0.60$ indicated moderate, $0.21-0.40$ indicated fair, and $0.01-$ 0.20 indicated poor. Analysis of the relationship between SEE, ITLPE, FIPE, and myometrial invasion based on histopathologic findings was performed with the $\chi^{2}$ or Fisher's exact test. According to the histopathologic findings, the sensitivity and specificity of SEE and ITLPE for myometrial invasion were calculated. The diagnostic performance of SEE and ITLPE for myometrial invasion was assessed by area under the curve (AUC) of the receiver operator characteristic (ROC) curve. The differences in sensitivity and specificity between SEE and ITLPE in evaluating myometrial invasion were analyzed with McNemar's test. A two-tailed $P$ value of $<0.05$ was considered statistically significant.

\section{RESULTS}

\section{MRI Findings}

Radiologist 1 identified 10/77 (12.9\%) patients with SEE, 55/77 (71.4\%) patients with ITLPE and 27/77 (35.1\%) patients with FIPE, whereas Radiologist 2 identified 14/77 (18.2\%) patients with SEE, 51/77 (66.2\%) patients with ITLPE and 32/77 (41.6\%) patients with FIPE. Inter-observer agreement was good $(k=0.80$;

TABLE 1 | MRI protocol: sequences and parameters.

\begin{tabular}{|c|c|c|c|c|c|}
\hline Sequence & Scanning plane & Repetition time (TR)/Echo time (TE) (ms) & Matrix size & Slice thickness/Gap (mm) & Field of view (mm) \\
\hline T2WI-TSE & Sagittal & $2,500 / 120$ & $280 \times 308$ & 6/0.6 & $250 \times 278$ \\
\hline T2WI-TSE & Axial & $3,000 / 110$ & $268 \times 253$ & $4 / 0.5$ & $240 \times 240$ \\
\hline T1WI-mDIXON & Axial & $5.8 / 1.8$ & $224 \times 175$ & $3 / 0$ & $400 \times 317$ \\
\hline $\mathrm{EPI}\left(\mathrm{b}=0,1,000 \mathrm{~s} / \mathrm{mm}^{2}\right)$ & Axial & $3,659 / 84$ & $144 \times 110$ & $6 / 0.6$ & $400 \times 300$ \\
\hline DCE-T1WI-mDIXON & Sagittal & $5.8 / 1.73$ & $188 \times 188$ & $2.5 / 0$ & $300 \times 300$ \\
\hline
\end{tabular}



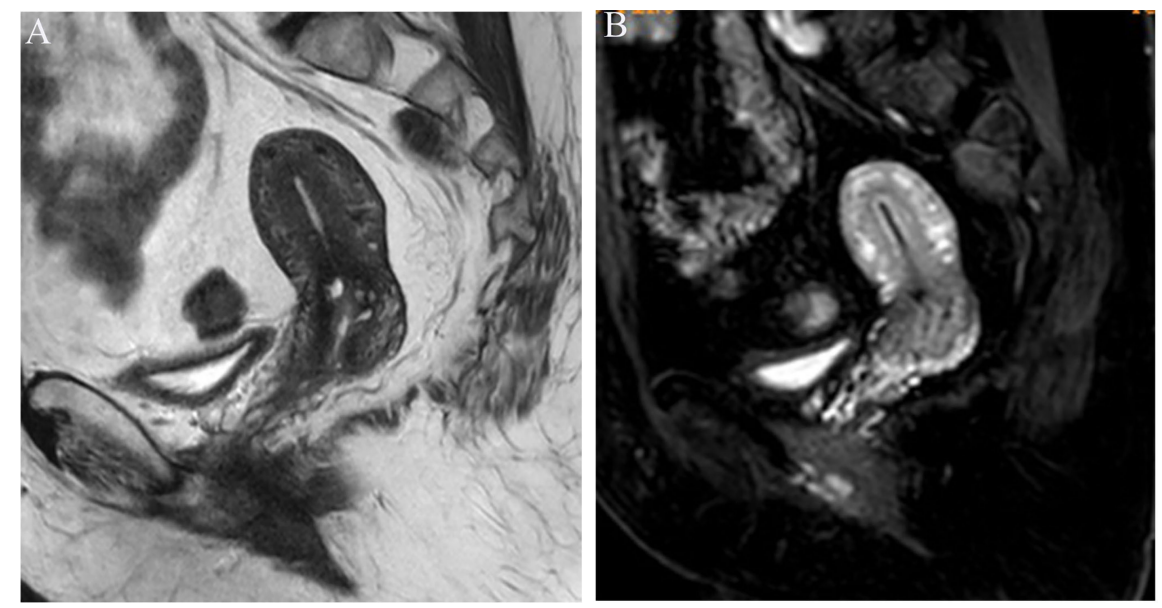

FIGURE 1 | (A) Sagittal-T2WI image, no definitive lesion is found in the uterine cavity. (B) Early (25.1 s) sagittal-DCE image shows the SEE, a thin enhancement layer between the endometrium and myometrium that is regular and smooth. This case was histologically proven to have endometrioid carcinoma, G1 with no myometrial invasion.

95\% CI, $0.577-0.955)$ for SEE, and very good $(k=0.88$; $95 \% \mathrm{CI}$, $0.761-0.972)(k=0.86 ; 95 \% \mathrm{CI}, 0.739-0.973)$ for ITLPE and FIPE. After consensus, SEE was identified in 12/77 (15.6\%) patients. ITLPE and FIPE were found in 53/77 (68.8\%) and 30/ $77(39.0 \%)$ patients, respectively. A statistically significant relationship was found between SEE, ITLPE, and myometrial infiltration $(P=0.000)$, but not FIPE $(P=0.725)$. The detailed information is shown in Table 2.

The sensitivity and specificity of SEE and ITLPE for diagnosing myometrial invasion in patients with low-risk endometrial carcinoma are shown in Table 3. The AUC values of SEE and
ITLPE for diagnosing myometrial invasion were 0.740 (95\% CI, $0.584-0.896$ ) and 0.866 (95\% CI, 0.763-0.970), respectively (Figure 4). Eleven cases were misdiagnosed by SEE, 8 cases were overestimated, and 3 cases were underestimated. For the overestimated cases, SEE was recognized as incomplete by the presence of FIPE in 6 cases (Figure 3) and ITLPE in 2 cases (Figure 5). For the underestimated cases, complete SEE seemed to be visible despite the presence of myometrial infiltration. Similarly, 11 cases were misdiagnosed based on ITLPE, 9 cases were underestimated and 2 cases were overestimated. ITLPE could not be identified with or without the presence of FIPE for the
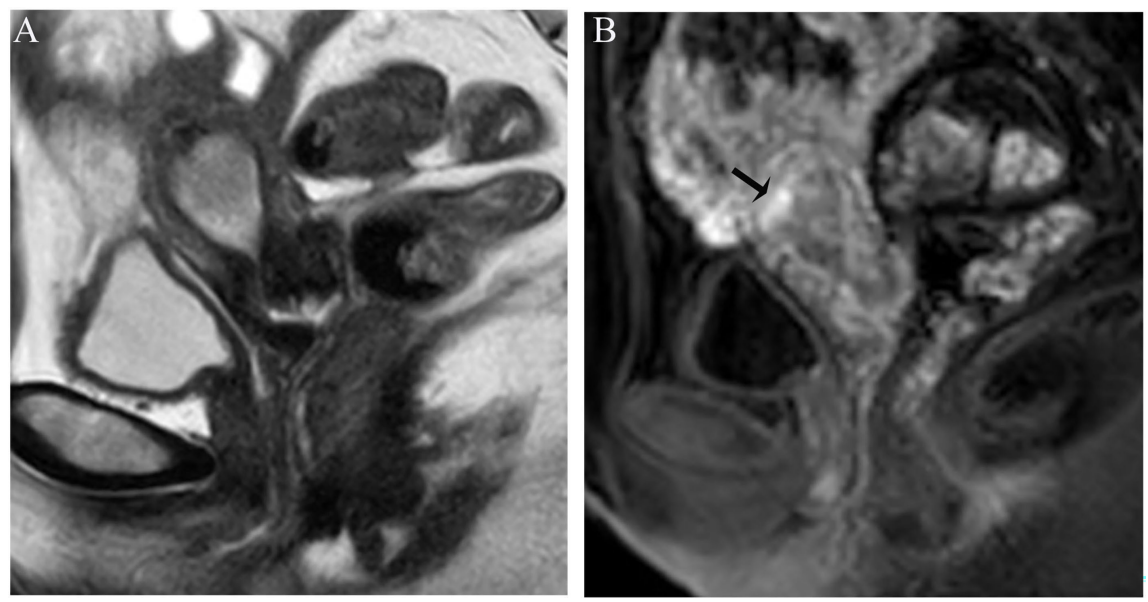

FIGURE 2 | (A) Sagittal-T2WI image, the tumor has moderate signal intensity and is found in the uterine cavity. (B) Early (32.9 s) sagittal-DCE image shows an irregular thin-layered enhancement (ITLPE); an irregular thin-layered enhancement in front of the tumor (black arrow). This case was histologically proven to have endometrioid carcinoma, G2 with superficial myometrial invasion. 

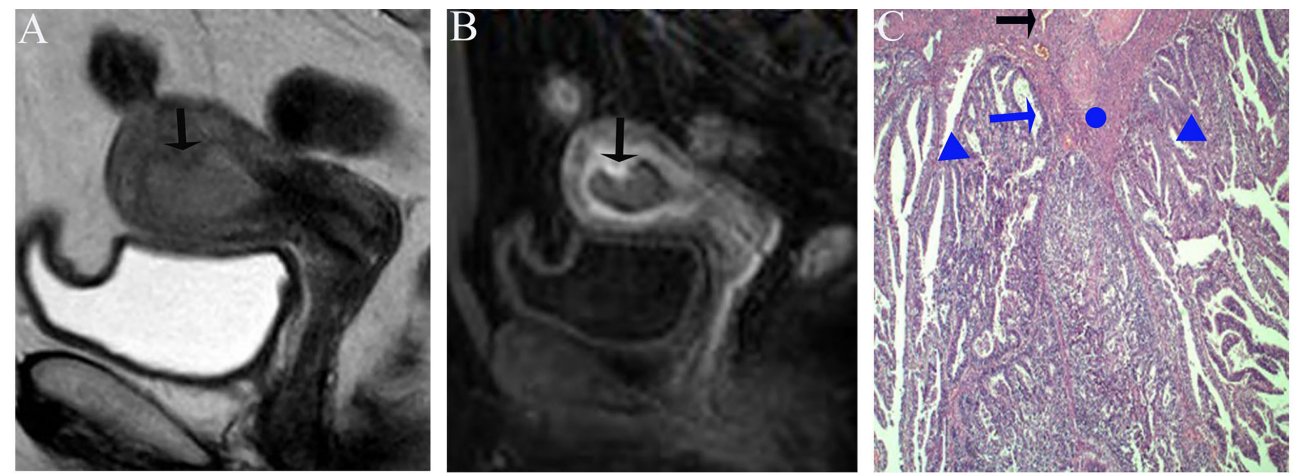

FIGURE 3 | (A) Sagittal-T2WI image, the tumor has moderate signal intensity and is found in the uterine cavity. The focal myometrium protrudes toward the lesion (black arrow). (B) Early (25.1 s) sagittal-DCE image shows the FIPE, a focal irregular enhancement (black arrow). (C) The photomicrograph (HE, 200x) shows the irregular endomyometrial junction (blue arrow) with the dilated vessels (black arrow) of the myometrium (circle). Note the undulating contour and extension of the myometrium between the tumors (triangle). This case was histologically proven to have endometrioid carcinoma, G1 with no myometrial invasion, and was overestimated by SEE.

underestimated cases (Figure 6). For the overestimated cases, ITLPE seemed to be visible despite the tumor being confined to the endometrium.

\section{Pathologic Findings and Comparison}

The histopathologic subtype of these 77 endometrial carcinoma cases was endometrioid adenocarcinoma; 17/77 (22.1\%) had no myometrial infiltration, and 60/77 (77.9\%) had superficial myometrial infiltration. In all, 41/77 (53.2\%) tumors were classified as grade 1 and $36 / 77(46.8 \%)$ tumors as grade 2 . Based on these histopathologic characteristics, all patients were classified as low-risk.

According to the comparison between FIPE and the histopathologic results, irregular endomyometrial junction can be found in $30 / 77$ (38.9\%) patients, $24 / 30$ (80.0\%) with myometrial infiltration, and 6/30 (20.0\%) without myometrial infiltration (Figures 3, 7).

\section{DISCUSSION}

Over recent years, DCE-MRI has been widely used in gynecological tumors, especially in the assessment of myometrial invasion in endometrial carcinoma $(31,32)$. The superior spatial and temporal resolution of DCE-MRI allowed us to observe more detailed information about the interface between the tumor and myometrium, such as ITLPE and FIPE. Our study demonstrated that the sensitivity and specificity for detecting myometrial invasion using SEE and ITLPE on DCE-MRI were 95.0, 52.9\% and 85.0, $88.0 \%$, respectively. Fujii et al. reported values of $96.6 \%$, 32.1-46.4\%, respectively, by using SEE (26). In our study, the specificity of ITLPE was higher than SEE, and higher than that of Fujii et al. In addition, in our results, the diagnostic performance of ITLPE for myometrial invasion in low-risk endometrial carcinoma was higher compared with SEE. Therefore, ITLPE that presented with high diagnostic performance and specificity maybe an efficient method to help younger patients avoid unnecessary hysterectomy. However, for the misdiagnosed cases, the main reason was that ITLPE seemed to be difficult to identify. Further temporal and spatial resolution improvements on DCE-MRI might enable more confident detection of ITLPE in future studies. Radiologists and gynecologists need the accurate identification of ITLPE to improve the diagnostic efficiency and specificity of myometrial infiltration.

The specificity of SEE for myometrial invasion was low in both our study and that of Fujii et al. The primary reason for the lower specificity was that we did not fully realize the nature of FIPE; thus, the presence of FIPE caused SEE to be misrecognized as incomplete. The comparison between the images and histopathologic findings indicated that FIPE was actually irregular endomyometrial junction caused by carcinomatous

TABLE 2 | Correlation between SEE, FIPE, ITLPE, and myometrial invasion.

\begin{tabular}{|c|c|c|c|c|c|c|}
\hline & \multicolumn{2}{|c|}{ SEE } & \multicolumn{2}{|c|}{ FIPE } & \multicolumn{2}{|c|}{ ITLPE } \\
\hline & $(+)$ & $(-)$ & $(+)$ & $(-)$ & $(+)$ & $(-)$ \\
\hline \multicolumn{7}{|c|}{ Myometrial invasion } \\
\hline$(+)$ & 3 & 57 & 24 & 36 & 51 & 9 \\
\hline$(-)$ & 9 & 8 & 6 & 11 & 2 & 15 \\
\hline$P$ & \multicolumn{2}{|c|}{0.000} & \multicolumn{2}{|c|}{0.725} & \multicolumn{2}{|c|}{0.000} \\
\hline
\end{tabular}


TABLE 3 | Diagnostic performance of SEE and ITLPE.

\begin{tabular}{lcc}
\hline $\mathbf{n}=\mathbf{7 7}$ & Sensitivity (\%) & Specificity (\%) \\
\hline SEE & $95.0(57 / 60)$ & $52.9(9 / 17)$ \\
ITLPE & $85.0(51 / 60)$ & $88.0(15 / 17)$ \\
$P$ & 0.031 & 0.016 \\
\hline
\end{tabular}

overgrowth without myometrial invasion. The irregular endomyometrial junction lent the appearance that the myometrium protruded toward the tumor with peripherally dilated vessels, which were found on the histopathologic tissue section (29). Ali et al. (30)reported that irregular endomyometrial junction was found in $57 \%$ of the surgical specimens of endometrial carcinoma. In our study, irregular endomyometrial junction was found in patients with or without myometrial infiltration, which was consistent with the study by Ali et al. In addition, our statistical analysis showed no association between FIPE and myometrial invasion. Therefore, FIPE should be taken into consideration in assessment of myometrial infiltration in lowrisk endometrial carcinoma by only using SEE.

Previous reports have not recommended MRI for the surgical staging of endometrial carcinoma because of the poor-tomoderate accuracy in detecting high-risk factors, namely, deep myometrial infiltration and cervical stromal invasion $(33,34)$. However, ESMO, the European Society for Radiotherapy (ESTRO) \& Oncology and the European Society of Gynaecological Oncology (ESGO) consensus conference on

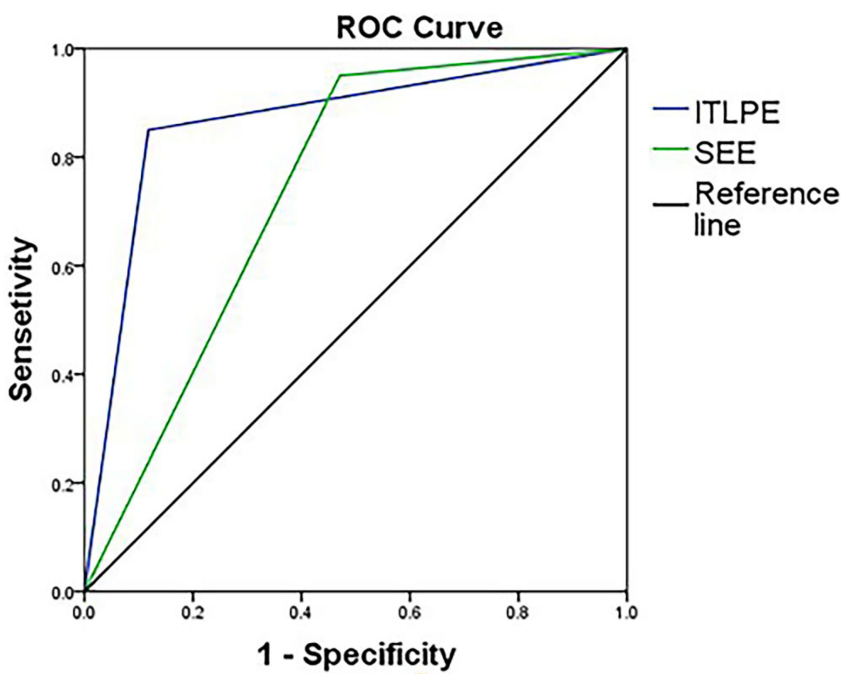

FIGURE 4 | Receiver operating characteristic curve of SEE and ITLPE for myometrial invasion in patients with low-risk endometrial carcinoma.
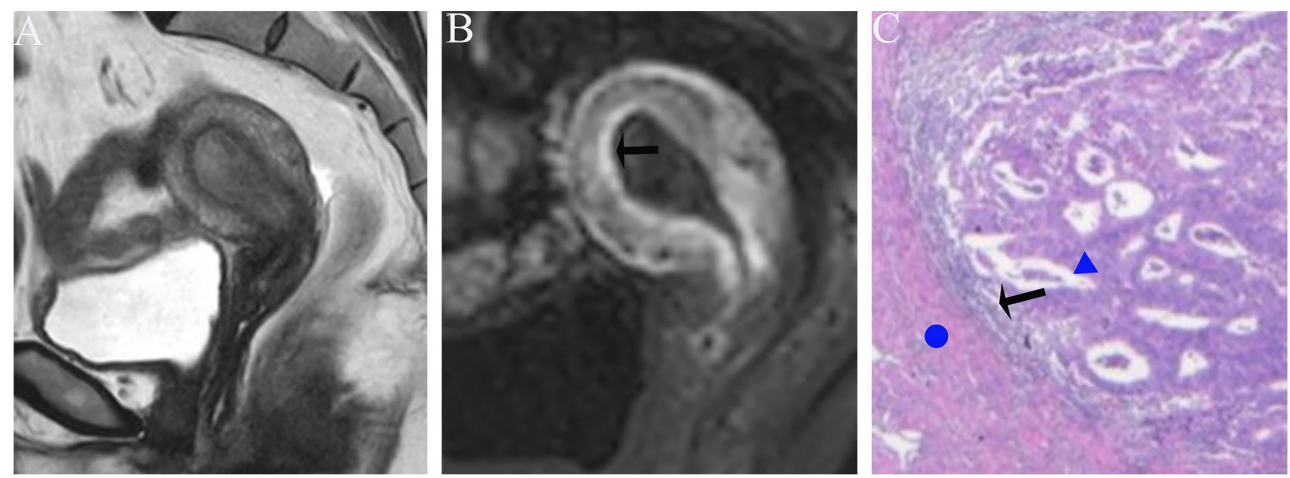

FIGURE 5 | (A) Sagittal-T2WI image, the tumor has moderate signal intensity and is found in the uterine cavity. (B) Early (32.9 s) sagittal-DCE image shows that the ITLPE seem to be visible at the anterior myometrium (black arrow). (C) A low-power photomicrograph (HE, 40x) shows the presence of endometrial stroma components (black arrow) between the tumor (triangle) and myometrium (circle). This case was histologically proven to have endometrioid carcinoma, G2 with no myometrial invasion, and was overestimated by SEE. 

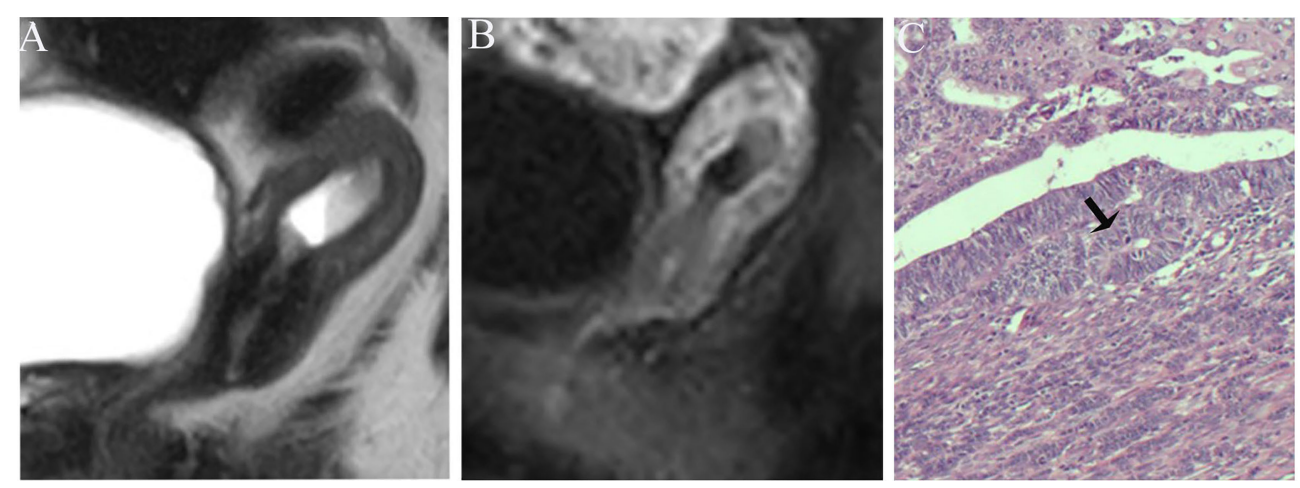

FIGURE 6 | (A) Sagittal-T2WI image, the tumor has moderate signal intensity and is found at the bottom of the uterus. (B) Early (32.9 s) sagittal-DCE image shows that the ITLPE seem not to be visible. (C) The photomicrograph (HE, 200x) shows that the tumor is surrounded by myometrium without intervening endometrial stroma components (black arrow). This case was histologically proven to have endometrioid carcinoma, G1 with superficial myometrial invasion, and is underestimated by ITLPE.
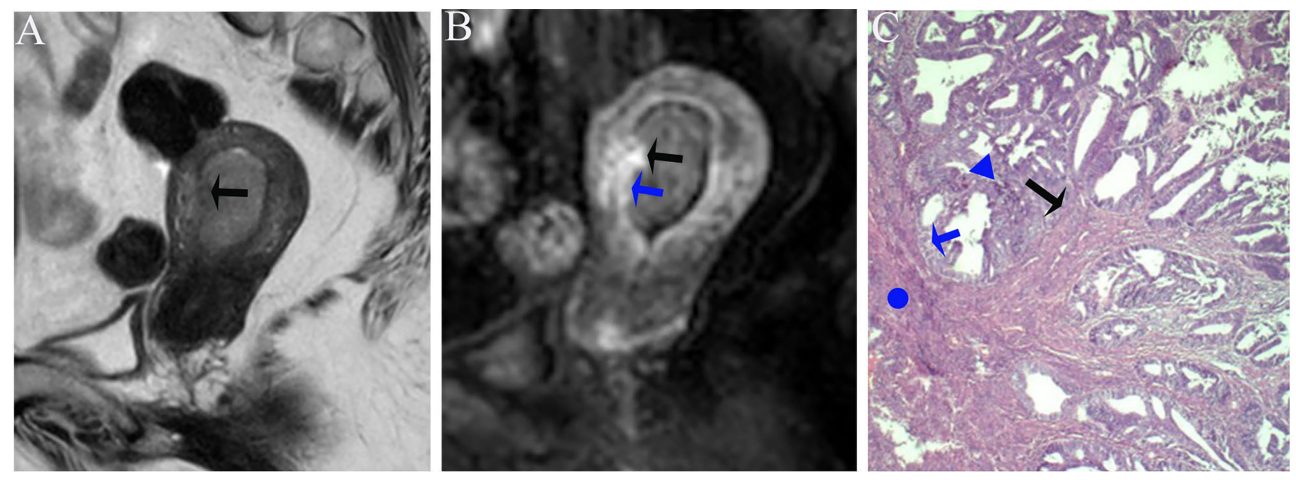

FIGURE 7 | (A) Sagittal-T2WI image, the tumor has moderate signal intensity and is found in the uterine cavity with focal myometrium protruding toward the lesion (black arrow). (B) Early (25.1 s) sagittal-DCE image shows the FIPE (black arrow) and ITLPE (blue arrow). (C) The photomicrograph (HE, 200x) shows that the tumor (triangle) is surrounded by the myometrium (circle) without intervening endometrial stroma components (blue arrow), but the irregular endomyometrial junction (undulating contour) can be found (black arrow). This case was histologically proven to have endometrioid carcinoma, G2 with superficial myometrial invasion.

endometrial cancer (9) indicated that MRI was preferred method for detecting tumors confined to the endometrium in patients with low-risk endometrial carcinoma who might have a chance to undergo fertility-sparing progestogen therapy. In clinical practice, radiologists and gynecologists should take FIPE into account when assessing myometrial infiltration by using SEE on DCE-MRI. Then, they should look for ITLPE, which may show evidence of myometrial infiltration when an intact SEE is not detected.

There are some limitations in our study. First, the sample size is relatively small, especially regarding the patients without myometrial invasion due to its low incidence $(22.1 \%)$. Secondly, the age range of patients was large (40-77 years), and some patients were postmenopausal. These factors may lead to bias in the diagnostic performance of low-risk endometrial carcinoma. Further studies should be performed in a large sample of young premenopausal patients with further improvement of the temporal and spatial resolution on DCE-MRI.

\section{DATA AVAILABILITY STATEMENT}

The original contributions presented in the study are included in the article/supplementary material. Further inquiries can be directed to the corresponding author.

\section{ETHICS STATEMENT}

The studies involving human participants were reviewed and approved by Beijing Shijitan Hospital, Capital Medical 
University. Written informed consent for participation was not required for this study in accordance with the national legislation and the institutional requirements. Written informed consent was not obtained from the individual(s) for the publication of any potentially identifiable images or data included in this article.

\section{REFERENCES}

1. Sung H, Ferlay J, Siegel RL, Laversanne M, Soerjomataram I, Jemal A, et al. Global Cancer Statistics 2020: GLOBOCAN Estimates of Incidence and Mortality Worldwide for 36 Cancers in 185 Countries. CA Cancer J Clin (2021) 71(3):209-49. doi: 10.3322/caac.21660

2. Raglan O, Kalliala I, Markozannes G, Cividini S, Gunter MJ, Nautiyal J, et al. Risk Factors for Endometrial Cancer: An Umbrella Review of the Literature. Int J Cancer (2019) 145(7):1719-30. doi: 10.1002/ijc.31961

3. Moore K, Brewer MA. Endometrial Cancer: Is This a New Disease? Am Soc Clin Oncol Educ Book (2017) 37:435-42. doi: 10.1200/edbk_175666

4. Soliman PT, Oh JC, Schmeler KM, Sun CC, Slomovitz BM, Gershenson DM, et al. Risk Factors for Young Premenopausal Women With Endometrial Cancer. Obstet Gynecol (2005) 105(3):575-80. doi: 10.1097/01.AOG.0000154151.14516.f7

5. Burleigh A, Talhouk A, Gilks CB, McAlpine JN. Clinical and Pathological Characterization of Endometrial Cancer in Young Women: Identification of a Cohort Without Classical Risk Factors. Gynecol Oncol (2015) 138(1):141-6. doi: 10.1016/j.ygyno.2015.02.028

6. Uharcek P, Mlyncek M, Ravinger J, Matejka M. Prognostic Factors in Women 45 Years of Age or Younger With Endometrial Cancer. Int J Gynecol Cancer (2008) 18(2):324-8. doi: 10.1111/j.1525-1438.2007.00997.x

7. Navarria I, Usel M, Rapiti E, Neyroud-Caspar I, Pelte MF, Bouchardy C, et al. Young Patients With Endometrial Cancer: How Many Could be Eligible for Fertility-Sparing Treatment? Gynecol Oncol (2009) 114(3):448-51. doi: 10.1016/j.ygyno.2009.05.038

8. Garg K, Soslow RA. Endometrial Carcinoma in Women Aged 40 Years and Younger. Arch Pathol Lab Med (2014) 138(3):335-42. doi: 10.5858/arpa.20120654-RA

9. Colombo N, Creutzberg C, Amant F, Bosse T, González-Martín A, Ledermann J, et al. ESMO-ESGO-ESTRO Consensus Conference on Endometrial Cancer: Diagnosis, Treatment and Follow-Up. Int J Gynecol Cancer (2016) 26(1):2-30. doi: 10.1097/igc.0000000000000609

10. Trojano G, Olivieri C, Tinelli R, Damiani GR, Pellegrino A, Cicinelli E. Conservative Treatment in Early Stage Endometrial Cancer: A Review. Acta BioMed (2019) 90(4):405-10. doi: 10.23750/abm.v90i4.7800

11. Harris KL, Maurer KA, Jarboe E, Werner TL, Gaffney D. LVSI Positive and NX in Early Endometrial Cancer: Surgical Restaging (and No Further Treatment If N0), or Adjuvant ERT? Gynecol Oncol (2020) 156(1):243-50. doi: 10.1016/j.ygyno.2019.09.016

12. Ebina Y, Katabuchi H, Mikami M, Nagase S, Yaegashi N, Udagawa Y, et al. Japan Society of Gynecologic Oncology Guidelines 2013 for the Treatment of Uterine Body Neoplasms. Int J Clin Oncol (2016) 21(3):419-34. doi: 10.1007/ s10147-016-0981-1

13. Amant F, Mirza MR, Koskas M, Creutzberg CL. Cancer of the Corpus Uteri. Int J Gynaecol Obstet (2018) 143 Suppl 2:37-50. doi: 10.1002/ijgo.12612

14. Yamazawa K, Seki K, Matsui H, Kihara M, Sekiya S. Prognostic Factors in Young Women With Endometrial Carcinoma: A Report of 20 Cases and Review of Literature. Int J Gynecol Cancer (2000) 10(3):212-22. doi: 10.1046/ j.1525-1438.2000.010003212.x

15. Arora V, Quinn MA. Endometrial Cancer. Best Pract Res Clin Obstet Gynaecol (2012) 26(3):311-24. doi: 10.1016/j.bpobgyn.2011.12.007

16. Lee NK, Cheung MK, Shin JY, Husain A, Teng NN, Berek JS, et al. Prognostic Factors for Uterine Cancer in Reproductive-Aged Women. Obstet Gynecol (2007) 109(3):655-62. doi: 10.1097/01.Aog.0000255980.88205.15

17. Colombo N, Preti E, Landoni F, Carinelli S, Colombo A, Marini C, et al. Endometrial Cancer: ESMO Clinical Practice Guidelines for Diagnosis,

\section{AUTHOR CONTRIBUTIONS}

TC, FS, and YY designed the study. CZ and JR collected the data. TC and JG analyzed the data. BG, YJ, and FS reviewed the data and interpreted the statistical analysis. TC drafted the manuscript. All authors contributed to the article and approved the submitted version.

Treatment and Follow-Up. Ann Oncol (2013) 24 Suppl 6:vi33-8. doi: 10.1093/annonc/mdt353

18. Haldorsen IS, Salvesen HB. Staging of Endometrial Carcinomas With MRI Using Traditional and Novel MRI Techniques. Clin Radiol (2012) 67(1):2-12. doi: 10.1016/j.crad.2011.02.018

19. Scoutt LM, McCarthy SM, Flynn SD, Lange RC, Long F, Smith RC, et al. Clinical Stage I Endometrial Carcinoma: Pitfalls in Preoperative Assessment With MR Imaging. Work in Progress. Radiology (1995) 194(2):567-72. doi: 10.1148/radiology.194.2.7824739

20. Seki H, Kimura M, Sakai K. Myometrial Invasion of Endometrial Carcinoma: Assessment With Dynamic MR and Contrast-Enhanced T1-Weighted Images. Clin Radiol (1997) 52(1):18-23. doi: 10.1016/s0009-9260(97)80300-5

21. Ito K, Matsumoto T, Nakada T, Nakanishi T, Fujita N, Yamashita H. Assessing Myometrial Invasion by Endometrial Carcinoma With Dynamic MRI. J Comput Assist Tomogr (1994) 18(1):77-86. doi: 10.1097/00004728-199401000-00017

22. Sironi S, Taccagni G, Garancini P, Belloni C, DelMaschio A. Myometrial Invasion by Endometrial Carcinoma: Assessment by MR Imaging. AJR Am J Roentgenol (1992) 158(3):565-9. doi: 10.2214/ajr.158.3.1738995

23. Lin G, Huang YT, Chao A, Ng KK, Yang LY, Ng SH, et al. Influence of Menopausal Status on Diagnostic Accuracy of Myometrial Invasion in Endometrial Cancer: Diffusion-Weighted and Dynamic Contrast-Enhanced MRI at 3 T. Clin Radiol (2015) 70(11):1260-8. doi: 10.1016/j.crad.2015.06.097

24. Nakao Y, Yokoyama M, Hara K, Koyamatsu Y, Yasunaga M, Araki Y, et al. MR Imaging in Endometrial Carcinoma as a Diagnostic Tool for the Absence of Myometrial Invasion. Gynecol Oncol (2006) 102(2):343-7. doi: 10.1016/ j.ygyno.2005.12.028

25. Horváth K, Pete I, Vereczkey I, Dudnyikova A, Gödény M. Evaluation of the Accuracy of Preoperative MRI in Measuring Myometrial Infiltration in Endometrial Carcinoma. Pathol Oncol Res (2014) 20(2):327-33. doi: 10.1007/s12253-013-9699-9

26. Fujii S, Kido A, Baba T, Fujimoto K, Daido S, Matsumura N, et al. Subendometrial Enhancement and Peritumoral Enhancement for Assessing Endometrial Cancer on Dynamic Contrast Enhanced MR Imaging. Eur J Radiol (2015) 84(4):581-9. doi: 10.1016/j.ejrad.2015.01.004

27. Yamashita Y, Harada M, Sawada T, Takahashi M, Miyazaki K, Okamura H. Normal Uterus and FIGO Stage I Endometrial Carcinoma: Dynamic Gadolinium-Enhanced MR Imaging. Radiology (1993) 186(2):495-501. doi: 10.1148/radiology.186.2.8421757

28. Fujii S, Kido A, Mikami Y, Matsumura N, Konishi I, Togashi K. Peritumoral Enhancement in Endometrial Cancer on Dynamic Contrast-Enhanced Imaging: Radiologic-Pathologic Correlation. J Obstet Gynaecol Res (2014) 40(5):1445-9. doi: 10.1111/jog.12318

29. Cole AJ, Quick CM. Patterns of Myoinvasion in Endometrial Adenocarcinoma: Recognition and Implications. Adv Anat Pathol (2013) 20(3):141-7. doi: 10.1097/PAP.0b013e31828d17cc

30. Ali A, Black D, Soslow RA. Difficulties in Assessing the Depth of Myometrial Invasion in Endometrial Carcinoma. Int J Gynecol Pathol (2007) 26(2):11523. doi: 10.1097/01.pgp.0000233165.56385.0b

31. Nougaret S, Horta M, Sala E, Lakhman Y, Thomassin-Naggara I, Kido A, et al. Endometrial Cancer MRI Staging: Updated Guidelines of the European Society of Urogenital Radiology. Eur Radiol (2019) 29(2):792-805. doi: 10.1007/s00330-018-5515-y

32. Himoto Y, Lakhman Y, Fujii S, Morita S, Mueller JJ, Leitao MMJr., et al. Multiparametric Magnetic Resonance Imaging Facilitates the Selection of Patients Prior to Fertility-Sparing Management of Endometrial Cancer. Abdom Radiol (NY) (2021) 46(9):4410-9. doi: 10.1007/s00261-021-03050-7 
33. Vandecaveye V, Dresen R, De Keyzer F. Novel Imaging Techniques in Gynaecological Cancer. Curr Opin Oncol (2017) 29(5):335-42. doi: 10.1097/ cco.0000000000000385

34. Luomaranta A, Leminen A, Loukovaara M. Magnetic Resonance Imaging in the Assessment of High-Risk Features of Endometrial Carcinoma: A MetaAnalysis. Int J Gynecol Cancer (2015) 25(5):837-42. doi: 10.1097/igc. 0000000000000194

Conflict of Interest: The authors declare that the research was conducted in the absence of any commercial or financial relationships that could be construed as a potential conflict of interest.
Publisher's Note: All claims expressed in this article are solely those of the authors and do not necessarily represent those of their affiliated organizations, or those of the publisher, the editors and the reviewers. Any product that may be evaluated in this article, or claim that may be made by its manufacturer, is not guaranteed or endorsed by the publisher.

Copyright $\odot 2022$ Cui, Shi, Gu, Jin, Guo, Zhang, Ren and Yue. This is an open-access article distributed under the terms of the Creative Commons Attribution License (CC BY). The use, distribution or reproduction in other forums is permitted, provided the original author(s) and the copyright owner(s) are credited and that the original publication in this journal is cited, in accordance with accepted academic practice. No use, distribution or reproduction is permitted which does not comply with these terms. 